\title{
Impactos ambientales generados por la mina de arcilla Wajira S.A.S., en Manaure, La Guajira
}

Daniel Cotes', Laura Alvarado², Edrianis Hoyos ${ }^{3}$, Marcela Molina ${ }^{4}$, Dayeli Mosquera ${ }^{5}$

\section{Resumen}

Las problemáticas ambientales más frecuentes en las minas de arcilla en el departamento de La Guajira se ven generalizadas por la afectación y modificación morfológica del suelo, atrasando la génesis del suelo, la tala de árboles, la pérdida de la capa vegetal, la erosión del suelo, la pérdida de hábitat en términos de fauna y problemas futuros en términos de $\mathrm{O}_{2}$, pues son estas plantas las que disminuyen el $\mathrm{CO}_{2}$ de la atmósfera para convertirlo en $\mathrm{O}_{2}$. La finalidad de este estudio es identificar los impactos ambientales generados por la mina de arcilla Wajira S. A. S. en Manaure, La Guajira. Este estudio está enmarcado en el tipo de investigación descriptivo con enfoque cualitativo, ya que, a través de valoraciones subjetivas, se realizará un diagnóstico de los impactos ambientales que genera la mina de arcilla Wajira S.A.S. Se concluye que la mayoría de los componentes, tal como el agua, el suelo, la flora (pérdida de biodiversidad y extinción de las especies endémicas), la fauna en la emigración de especies y disminución de las especies endémicas, estudiadas para las fases de construcción y operación, generan impacto moderado, es decir, requieren medidas bien elaboradas. La recuperación de las condiciones iniciales ameritan cierto tiempo y se precisan prácticas de manejo simples.

Palabras clave: arcilla, impacto ambiental, lista de chequeo.

\section{Introducción}

En Colombia la deforestación es el principal disturbio antrópico que afecta a todos los ecosistemas terrestres y algunos costeros; la destrucción y los cambios en la 
cobertura vegetal son causa directa de la pérdida de biodiversidad. Las actividades que mayor deforestación generan en los bosques tropicales son la extracción selectiva de maderas de gran valor económico, el establecimiento de sistemas de producción agrícolas y ganaderos y cultivos ilícitos, la explotación de minerales a cielo abierto, la expansión urbana, la construcción de obras de infraestructura, entre ellas carreteras y embalses, así como la extracción de leña para combustible y cercas vivas, cuyo impacto es a menor escala que todas las anteriores, y todo esto debido a que no existe una adecuada planificación ambiental que permita el control y prevenga todos estos impactos causados. (Etter, 1998 citado en IDEAM y MAVDS, 2011)

La puesta en marcha de una actividad minera genera contaminantes $y$ sólidos, que de una forma u otra van a parar al suelo, esto e presenta ya sea por depósito a partir de la atmósfera como partículas sedimentadas o traídas por las aguas de lluvia, por el vertido directo de los productos líquidos de la actividad minera, o por la infiltración de productos de lixiviación del entorno minero: aguas provenientes de minas a cielo abierto, escombreras, etc., o por la disposición de elementos mineros sobre el suelo: escombreras, talleres de la mina u otras edificaciones más o menos contaminantes en cada caso. (Vega, 2007)

La explotación a cielo abierto puede desarrollar: perdida de cobertura vegetal que ocupa un área específica dentro de un ecosistema, este cumple funciones como la captación y almacenamiento de energía, refugio de la fauna, y previene procesos erosivos del suelo, etc. Además, representa una modificación radical de la morfología; condiciones de inestabilidad; sedimentación de cauces por arrastre de materiales y escorrentía; intervención de áreas de recarga de acuíferos. (MAVDT, 2010)

“Contaminación por ruido, vibraciones y circulación de la maquinaria utilizada; emisión de partículas y gases a la atmósfera y deterioro de la infraestructura vial por tráfico de vehículos de carga" (Sánchez, 2001 citado por Garzón, 2013). Por consiguiente, se pueden generar afectaciones ocasionada en el proceso 
industrial de la producción de ladrillos y bloques, el cual se encuentra sujeto a una serie de actividades que requieren insumos naturales como el recurso hídrico, al que se debe aplicar las normas de buen uso. Cabe destacar que para este proceso se necesitan maquinarias $\mathrm{y}$ equipos que en sus salidas generan impactos, como son el tema de los gases en relación con la atmósfera y la salud de las personas que viven en zonas aledañas al sitio de producción, además los desechos o residuos sólidos y las aguas residuales que deben ser manejadas; por otro lado, en la actividad referente al tema de los hornos y el recurso que se utilice, como en el caso de la madera, es un problema que se debe considerar y planificar, ya que es un recurso finito, además, produce el efecto conocido como isla caliente y el uso irracional de este recurso afecta directamente las condiciones fisicoquímicas del suelo y genera algunas problemáticas sociales.

En la mina de arcilla Wajira S.A.S en Manaure, La Guajira, la explotación del material se realiza para la elaboración de productos ladrillos y bloques que van a satisfacer la demanda de la industria de la construcción, preferiblemente a los municipios que componen el departamento de La Guajira. Por lo anterior, se resalta que esta empresa maneja o emplea dos áreas de trabajo en las que, principalmente, se encuentra la actividad de extracción y obtención del material arcilloso por la minería y, en la otra, el proceso de producción de ladrillos y bloque mediante actividades industriales. Las problemáticas ambientales más frecuentes en las minas de arcilla en el departamento de La Guajira se ven generalizadas por la afectación y modificación morfológica del suelo, atrasando la génesis del suelo, la tala de árboles, la pérdida de la capa vegetal, la erosión del suelo, la pérdida de hábitat en términos de fauna y problemas futuros en términos de $\mathrm{O} 2$, pues son estas plantas las que disminuyen el $\mathrm{CO} 2$ de la atmósfera para convertirlo en $\mathrm{O} 2$.

Esta investigación se centra en el análisis de los impactos ambientales generados por la minería a cielo abierto en el municipio de Manaure, con la finalidad de identificar y buscar medidas de control, prevención, mitigación y de compensación por los impactos generados, buscando con ello la conservación y recuperación de áreas degradadas por estas actividades extractivas. Es bueno comprender que el crecimiento socioeconómico es elemental para una sociedad; principalmente como la de la región Caribe colombiano, ya que esta cuenta con una diversidad de minerales que 
pueden ser aprovechados de manera positiva, generando empleo y permitiendo un avance social; a su vez, la humanidad debe pensar en el desarrollo sostenible y que las generaciones futuras tienen derecho de disfrutar y vivir en un planeta con las comodidades ambientales necesarias.

\section{Metodología \\ Localización}

La Mina de Arcilla Wajira S.A.S. está ubicada en el kilómetro 7 vía Riohacha a Maicao, en el corregimiento de Paumahana, municipio de Manaure, La Guajira. Sus coordenadas son, $11^{\circ} 31^{\prime} 16.14$ ” N y 7250'39.18” O. En el área de interés, el material extraído son las arcillas. La zona del corregimiento de Paumahana, municipio de Manaure, se caracteriza por presentar temperaturas que oscilan entre $28^{\circ} \mathrm{C}$ y $35^{\circ} \mathrm{C}$, la temperatura se ve altamente influenciada por la intensa insolación característica de la zona. El área de estudio, en general, presenta una topografía plana, también cuenta con una gran variedad de especies arbóreas.

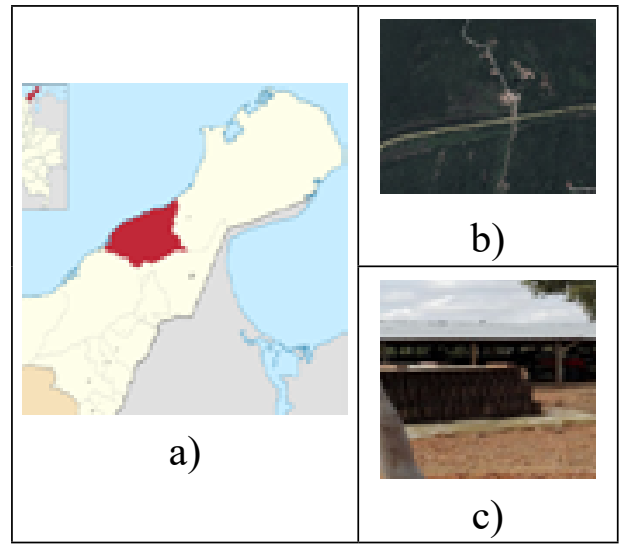

Figura 1. Localización del área de estudio

a) localización del municipio de Manaure, en La Guajira; b) ubicación del corregimiento de Pauhamana; y c) Mina de arcilla Wajira S.A.S.

\section{Tipo de investigación}

Este estudio está enmarcado en el tipo de investigación descriptivo con enfoque cualitativo, ya que a través de valoraciones subjetivas se realizará un diagnóstico de los impactos ambientales que genera la mina de arcilla Wajira S.A.S.

Para alcanzar los objetivos específicos se realizará observación, en la cual se identificará cuál es el proceso utilizado para la elaboración del ladrillo, así como también se identificarán los 
riesgos ambientales presentados en esta mina; además se realizarán entrevistas para contrastar la información vista en campo; también, se aplicarán listas de chequeo, las cuales son relaciones categorizadas o jerárquicas de factores ambientales a partir de las cuales se identifican los impactos producidos por un proyecto o actividad específica, con el fin de valorar los impactos ambientales presentados. Finalmente, se realizará un análisis de los resultados y se propondrán unas recomendaciones para mitigar dichos impactos.

\section{Resultados}

Riesgos ambientales presentados en la mina de arcilla Wajira S. A.S., en Manaure, La Guajira

En la mina Wajira S.A.S. existe un uso irracional de los recursos y materia prima utilizados en el proceso que realiza la mina de arcilla Wajira S.A.S. No se hallan procesos que generen una reducción del consumo energético,yaque, durante el desarrollo de la combustión, se incrementa la emisión de gases efecto invernadero que contribuyen de forma negativa al calentamiento global. Esto, a su vez, genera una emisión de material particulado a la atmósfera, tanto por la combustión como por la extracción y manipulación del material, lo cual puede ocasionar afectaciones a la salud como problemas en el sistema respiratorio. La recuperación de residuos sólidos en la técnica de fabricación aún no tiene un manejo eficiente, lo que puede generar pérdidas económicas como un costo excesivo en las actividades de producción y afectaciones al ambiente debido a las pérdidas de las condiciones naturales del suelo respecto a la flora y la fauna.

La toma indiscriminada de los recursos naturales tanto por parte de la comunidad como de la empresa, tales como la arcilla que se extrae directamente de las minas existentes en la zona, la arena de río, aserrín o arena fina (polvillo), agua y la madera como combustible; junto a ello son requeridos el recurso humano, los equipos y las herramientas al igual que los hornos para dar inicio al proceso; pueden producir acciones como la tala indiscriminada, disminución de la cobertura vegetal, deterioro del paisaje erosión del suelo y múltiples efectos más producto de dicha actividad. Es por ello que se requiere adoptar medidas que permitan mitigar los impactos negativos generados al medio ambiente. 


\section{Listas de chequeo. Control 0 verificación}

\section{Tabla 1. Lista de chequeo para identificar impactos ambientales en la mina de arcilla Wajira S.A.S.}

\begin{tabular}{|c|c|c|c|c|}
\hline \multirow{2}{*}{ Impactos generados } & \multicolumn{4}{|c|}{ Etapa del proyecto } \\
\hline & Diseño & Construcción & Operación & Abandono o cierre \\
\hline $\begin{array}{l}\text { 1. Sobre el aire } \\
\text { 1.1. Contaminación. } \\
\text { 1.2. Emisión de material } \\
\text { particulado } \\
\text { 1.3. Incremento de ruido }\end{array}$ & & $\begin{array}{l}\mathrm{x} \\
\mathrm{x}\end{array}$ & $\begin{array}{l}\mathrm{x} \\
\mathrm{x}\end{array}$ & $\mathrm{x}$ \\
\hline $\begin{array}{l}\text { 2. Sobre el suelo } \\
\text { 2.1. Pérdida de vegetación } \\
\text { 2.2. Pérdida de suelos } \\
\text { fértiles } \\
\text { 2.3. Erosión }\end{array}$ & & $\begin{array}{l}\mathrm{x} \\
\mathrm{x} \\
\mathrm{x}\end{array}$ & & $\mathrm{x}$ \\
\hline $\begin{array}{l}\text { 3. Sobre la vegetación } \\
\text { 3.1. Deforestación } \\
\text { 3.2. Pérdida de } \\
\text { biodiversidad } \\
\text { 3.3. Efecto sobre especies } \\
\text { endémicas }\end{array}$ & & $\begin{array}{l}\mathrm{x} \\
\mathrm{x} \\
\mathrm{x}\end{array}$ & & $\begin{array}{l}\mathrm{x} \\
\mathrm{x} \\
\mathrm{x}\end{array}$ \\
\hline $\begin{array}{l}\text { 4. Sobre la población } \\
\text { 4.1. Generación de em- } \\
\text { pleo }\end{array}$ & & $\mathrm{x}$ & $\mathrm{x}$ & \\
\hline $\begin{array}{l}\text { 5. Sobre el agua } \\
\text { 5.1. Contaminación de } \\
\text { acuíferos } \\
\text { 5.2. Cambio de uso }\end{array}$ & & $\begin{array}{l}\mathrm{x} \\
\mathrm{x}\end{array}$ & $\mathrm{x}$ & $\mathrm{x}$ \\
\hline $\begin{array}{l}\text { 6. Otros } \\
\text { 6.1. Pérdida del paisaje }\end{array}$ & & $\mathrm{x}$ & & $\mathrm{x}$ \\
\hline
\end{tabular}




\section{Causas de los problemas ambientales}

Pérdida de la cobertura vegetal

Corresponde al primer paso a llevar a cabo en casi todos los proyectos mineros a cielo abierto, consiste en despojar la zona de interés de la capa vegetal para poder acceder a los horizontes objeto de explotación, muy pocas veces en los frentes de explotación se recupera la cobertura vegetal, en algunos casos se realiza parcialmente después de terminar las labores mineras en un sitio determinado. La pérdida de cobertura desencadena con frecuencia procesos erosivos, a excepción de los lugares en los que aflora roca fresca, es decir, poco meteorizada.

\section{Erosión}

La erosión es el proceso natural de desgaste o destrucción de los suelos, pero para el caso de las prácticas mineras, la erosión se acelera, ya que como se mencionó arriba, es necesario retirar la capa vegetal y dejar el suelo o la roca expuesta. El avance de este proceso genera otros fenómenos como surcos, cárcavas y cuando no se toman ciertas medidas se generan deslizamientos, los cuales llegan a obstaculizar las labores mineras o afectar los barrios aledaños. (Alcaldía de Medellín, 2011)

\section{Afectación del paisajismo}

Este problema combina los dos problemas anteriores, ya que los lugares que han sido afectados por procesos mineros, presentan poca cobertura vegetal y generalmente son zonas afectadas por procesos erosivos. También es común observar cambios morfológicos como consecuencia de las actividades mineras y en muchos casos recuperar la cobertura vegetal es un proceso difícil y lento, ya que es frecuente que queden expuestas zonas poco meteorizadas y el proceso de revegetalización tarda más del tiempo normal. (Ramírez, 2008)

\section{Contaminación del aire}

La contaminación del aire está fuertemente relacionada a las zonas donde se presentan grandes superficies desprovistas de vegetación, ya que es habitual el levantamiento de partículas que son transportadas por el viento especialmente en las épocas de 
verano, generando grandes nubes de polvo en suspensión. (Ramírez, suelo 2008)

\section{Generación de ruido}

Este problema se presenta especialmente en la explotación del mineral donde se emplea maquinaria para estas labores, no solo por el uso de explosivos, sino también por el continuo golpe de las palas de las retroexcavadoras a los frentes de trabajo y el cargue de las volquetas que sacan el material para la venta. (Benito y Huamán, 2014)

\section{Plan de Manejo Ambiental}

Como programas y fichas consideradas para las operaciones del proyecto de la evaluación de impacto ambiental de la mina de arcilla Wajira S.A.S. en el municipio de Manaure, La Guajira son los siguientes:
I programa: mejorar la calidad del

- MCS 001. Restaurar la capacidad de producción del suelo (fertilidad).

II programa: manejo de material particulado

- MP 002. Medidas de control para el material particulado que afecta al personal.

III programa: plan de recuperación de la flora

- MRF 003. Medidas para la conservación de la flora.

IV programa: medidas de corrección y mitigación

- PGS 005. Determinación de la calidad de las aguas subterráneas y superficiales.

Seguido de esto, se presentarán las fichas de manejo ambiental aplicadas a cada uno de los programas elaborados para este estudio. 
Tabla 2. Ficha de prevención y mitigación para mejoramiento del suelo

\begin{tabular}{|c|c|c|c|}
\hline \multirow[b]{2}{*}{ Ficha } & \multirow[b]{2}{*}{ MCS 001} & Programa & $\begin{array}{l}\text { Mejorar la calidad del } \\
\text { suelo }\end{array}$ \\
\hline & & Proyecto & $\begin{array}{l}\text { Restaurar la capacidad de } \\
\text { producción del suelo }\end{array}$ \\
\hline Objetivo & \multicolumn{3}{|c|}{$\begin{array}{l}\text { Aplicar medidas para la sostenibilidad del suelo y minimizar la } \\
\text { disposición de material calcáreo en este. }\end{array}$} \\
\hline Meta & \multicolumn{3}{|c|}{ Soporte de las plantas } \\
\hline $\begin{array}{l}\text { Componentes am- } \\
\text { bientales afectados }\end{array}$ & $\begin{array}{ll}\text { - } & \text { Agua } \\
\text { - } & \text { Flora } \\
\text { - } & \text { Fauna } \\
\text { - } & \text { Suelo }\end{array}$ & $\begin{array}{l}\text { Aspectos } \\
\text { ambientales }\end{array}$ & $\begin{array}{l}\text { Deterioro de la calidad } \\
\text { del suelo }\end{array}$ \\
\hline $\begin{array}{l}\text { Actividades que oca- } \\
\text { sionan el impacto }\end{array}$ & \multicolumn{3}{|c|}{ - Extracción de arcilla } \\
\hline Efectos & \multicolumn{3}{|c|}{$\begin{array}{l}\text { Las excavaciones y tomas de arcilla del suelo generan pérdida } \\
\text { de componentes elementales para las características morfomé- } \\
\text { tricas del mismo. }\end{array}$} \\
\hline Impactos ambientales & \multicolumn{3}{|c|}{$\begin{array}{l}\text { Deterioro de la composición físicoquímica del } \\
\text { suelo ocasionando pérdidas de nutrientes para poder } \\
\text { abastecer a las plantas. } \\
\text { - Contaminación de cuerpos de aguas, por aguas con } \\
\text { sedimentos de material depositado. }\end{array}$} \\
\hline
\end{tabular}


Tabla 3. Tipos de medidas para la prevención y mitigación para mejoramiento del suelo

\begin{tabular}{|c|c|c|c|c|c|}
\hline \multicolumn{6}{|c|}{ Tipos de medidas: prevención y mitigación } \\
\hline Lugar de aplicación & \multicolumn{5}{|c|}{ Mina de Arcilla Wajira, S.A.S. } \\
\hline \multicolumn{6}{|c|}{ Acciones por desarrollar } \\
\hline \multicolumn{6}{|c|}{$\begin{array}{l}\text { - Mantenimiento continuo de las zonas de disposición de material depositado. } \\
\text { - } \quad \text { rar ladrillos comerciales. } \\
\text { - Construcción de canales de recolección y conducción de aguas de escorrentías. }\end{array}$} \\
\hline \multicolumn{6}{|c|}{ Alternativas para realizar las acciones planteadas } \\
\hline \multicolumn{6}{|c|}{$\begin{array}{l}\text { "El procedimiento de disposición de material calcáreo establece el método de construcción de la zona } \\
\text { de disposición del material. Se deben implementar desde las fases de la explotación los conceptos de } \\
\text { localización, diseño, construcción, manejo y adecuación de material calcáreo, para prevenir y mitigar } \\
\text { los impactos propios de la actividad" (Avancejuridico.com). }\end{array}$} \\
\hline \multicolumn{6}{|c|}{$\begin{array}{l}\text { Descripción de las medidas } \\
\end{array}$} \\
\hline \multicolumn{6}{|c|}{ Prevención y mitigación } \\
\hline \multicolumn{6}{|c|}{$\begin{array}{l}\text { Objetivo: compensar de manera ambiental el suelo teniendo en cuenta su uso en terrenos forestales, } \\
\text { en el que se tiene como propósito llevar a cabo acciones para la restauración de los suelos, refores- } \\
\text { tación y mantenimientos de los ecosistemas forestales. }\end{array}$} \\
\hline Actividades & $\begin{array}{l}\text { Tiempo de } \\
\text { ejecución } \\
\text { (mes) }\end{array}$ & Responsable & $\begin{array}{l}\text { Recurso } \\
\text { humano }\end{array}$ & $\begin{array}{l}\text { Recurso } \\
\text { físico }\end{array}$ & $\begin{array}{c}\text { Costos } \\
\text { asociados }\end{array}$ \\
\hline $\begin{array}{l}\text { Recuperación del } \\
\text { suelo a partir del } \\
\text { compost }\end{array}$ & 3 & \multirow[b]{2}{*}{$\begin{array}{l}\text { Experto en cali- } \\
\text { dad del suelo }\end{array}$} & 4 & $\begin{array}{l}\text { Abono, } \\
\text { cavadoras, } \\
\text { y palas }\end{array}$ & $\$ 2500000$ \\
\hline $\begin{array}{l}\text { Mantenimientos de } \\
\text { ecosistemas fores- } \\
\quad \text { tales }\end{array}$ & 3 & & 2 & $\begin{array}{l}\text { Folletos, } \\
\text { Pancartas, } \\
\text { avisos } \\
\text { viales }\end{array}$ & $\$ 2000000$ \\
\hline
\end{tabular}


Tabla 4. Ficha de mitigación para el control de material particulado

\begin{tabular}{|c|c|c|c|}
\hline \multirow[b]{2}{*}{ Ficha } & \multirow[b]{2}{*}{ MP 002} & Programa & $\begin{array}{l}\text { Manejo de emisión mate- } \\
\text { rial particulado }\end{array}$ \\
\hline & & Proyecto & $\begin{array}{l}\text { Medidas de control para el } \\
\text { material particulado pro- } \\
\text { ducido por la empresa que } \\
\text { afecta al personal. }\end{array}$ \\
\hline Objetivo & \multicolumn{3}{|c|}{$\begin{array}{l}\text { Mitigar los niveles de material particulado producidos por la extracción } \\
\text { del mineral, transporte, calcinación, descargue, y fabricación de ladri- } \\
\text { llos, alterando la calidad del aire y afectaciones a la flora. Mantener la } \\
\text { calidad del aire bajo los valores permisibles exigidos por la norma. }\end{array}$} \\
\hline Meta & \multicolumn{3}{|c|}{$\begin{array}{l}\text { "Cumplir con los niveles máximos permisibles de material particulado } \\
\text { de acuerdo con la norma vigente" (Avancejuridico.com). }\end{array}$} \\
\hline $\begin{array}{l}\text { Componentes ambienta- } \\
\text { les afectados }\end{array}$ & $\begin{array}{ll}- & \text { Aire } \\
\text { - } & \text { Flora } \\
\text { - } & \text { Fauna } \\
\text { - } & \text { Ser hu- } \\
& \text { mano }\end{array}$ & $\begin{array}{l}\text { Aspectos ambien- } \\
\text { tales }\end{array}$ & $\begin{array}{l}\text { Deterioro de la calidad del } \\
\text { aire y la salud humana. }\end{array}$ \\
\hline $\begin{array}{l}\text { Actividades que ocasio- } \\
\text { nan el impacto }\end{array}$ & \multicolumn{3}{|c|}{$\begin{array}{l}\text { - } \quad \text { Cargue del recurso explotado y transformado. } \\
\text { - } \quad \text { Transporte del recurso explotado y transformado. }\end{array}$} \\
\hline Efectos & \multicolumn{3}{|c|}{$\begin{array}{l}\text { En el cargue y transporte del recurso se emite material particulado, } \\
\text { causante de cambios en la atmósfera, calentamientos, problemas respi- } \\
\text { ratorios, visibilidad y edemas pulmonares. }\end{array}$} \\
\hline Impactos ambientales & \multicolumn{3}{|c|}{$\begin{array}{l}\text { - } \quad \text { Deterioro de la calidad del aire en la zona de influencia. } \\
\text { - } \quad \text { Afectación de la vegetación. } \\
\text { Disminución de la calidad paisajística por disminución de la } \\
\text { visibilidad. }\end{array}$} \\
\hline
\end{tabular}


Tabla 5. Tipos de medidas para el control de material particulado

\begin{tabular}{|c|c|c|c|c|c|}
\hline \multicolumn{6}{|c|}{ Tipos de medidas: mitigación } \\
\hline $\begin{array}{l}\text { Lugar de } \\
\text { aplicación }\end{array}$ & \multicolumn{5}{|c|}{ Mina de Arcilla Wajira, S.A.S. } \\
\hline \multicolumn{6}{|c|}{ Acciones por desarrollar } \\
\hline \multicolumn{6}{|c|}{$\begin{array}{l}\text { - "Realizar medidas de mitigación de emisión de partículas, como barreras rompe vientos } \\
\text { - En patios de acopio y cubrimiento de pilas de material. } \\
\text { - } \text { de material particulado. } \\
\text { Los vehículos que transportan material deben poseer carpas o protectores hechos de } \\
\text { material resistente debidamente asegurados para evitar escapes de desechos" (Avanceju- } \\
\text { ridico.com). }\end{array}$} \\
\hline \multicolumn{6}{|c|}{ Alternativas para realizar las acciones planteadas } \\
\hline \multicolumn{6}{|c|}{$\begin{array}{l}\text { - Construcción de barreras rompe vientos en las zonas donde exista mayor generación de } \\
\text { material particulado. } \\
\text { - Dispositivos protectores, carpas o cobertura para los vehículos. } \\
\text { Capacitación sobre las medidas de manejo de la emisión de material particulado"(Avan- } \\
\text { cejuridico.com). }\end{array}$} \\
\hline \multicolumn{6}{|c|}{ Descripción de las medidas } \\
\hline \multicolumn{6}{|c|}{ Mitigación } \\
\hline \multicolumn{6}{|c|}{$\begin{array}{l}\text { Objetivo: Minimizar las emisiones de material particulado en el área de influencia con el fin de } \\
\text { evitar la contaminación de la atmósfera y afectación a la salud de los seres vivos. }\end{array}$} \\
\hline Actividades & $\begin{array}{l}\text { Tiempo de } \\
\text { ejecución } \\
\text { (mes) }\end{array}$ & Responsable & $\begin{array}{l}\text { Recurso } \\
\text { humano }\end{array}$ & $\begin{array}{l}\text { Recurso } \\
\text { físico }\end{array}$ & $\begin{array}{c}\text { Costos } \\
\text { asociados }\end{array}$ \\
\hline $\begin{array}{l}\text { Sistema de asper- } \\
\text { sión }\end{array}$ & $\begin{array}{l}\text { Toda la vida } \\
\text { útil del pro- } \\
\text { yecto }\end{array}$ & \multirow{2}{*}{$\begin{array}{l}\text { Personal capa- } \\
\text { citado en el área } \\
\text { de mitigación de } \\
\text { partículas }\end{array}$} & 4 & $\begin{array}{l}\text { Bombeo, } \\
\text { tuberías y } \\
\text { camión }\end{array}$ & $\$ 2500000$ \\
\hline $\begin{array}{l}\text { Educación de con- } \\
\text { ductores }\end{array}$ & 3 & & 2 & $\begin{array}{l}\text { Folletos, } \\
\text { Pancartas, } \\
\text { avisos } \\
\text { viales }\end{array}$ & $\$ 2000000$ \\
\hline
\end{tabular}


Tabla 6. Ficha de mitigación para la conservación de la flora

\begin{tabular}{|c|c|c|c|}
\hline \multirow[t]{2}{*}{ Ficha } & \multirow[t]{2}{*}{ MRF 003} & Programa & $\begin{array}{l}\text { Plan de Recuperación } \\
\text { de la flora }\end{array}$ \\
\hline & & Proyecto & $\begin{array}{l}\text { Medidas para la con- } \\
\text { servación de la Flora }\end{array}$ \\
\hline Objetivo & \multicolumn{3}{|c|}{$\begin{array}{l}\text { Minimizar los impactos realizados sobre el sistema paisajís- } \\
\text { tico para la recuperación de flora endémica. }\end{array}$} \\
\hline Meta & \multicolumn{3}{|c|}{$\begin{array}{l}\text { La flora como fuente básica del ser vivo por la producción de } \\
\text { oxígeno. }\end{array}$} \\
\hline $\begin{array}{l}\text { Componentes Ambien- } \\
\text { tales afectados }\end{array}$ & $\begin{array}{ll}\text { - } & \text { Flora } \\
\text { - } & \text { Fauna } \\
\text { - } & \text { Social } \\
\text { Paisaje }\end{array}$ & $\begin{array}{l}\text { Aspectos am- } \\
\text { bientales }\end{array}$ & $\begin{array}{l}\text { Deterioro de la cali- } \\
\text { dad de la flora. }\end{array}$ \\
\hline $\begin{array}{l}\text { Actividades que oca- } \\
\text { sionan el impacto }\end{array}$ & \multicolumn{3}{|c|}{$\begin{array}{l}\text { - Tala de árboles presentes en la zona de estudio. } \\
\text { - Disposición de escombros. }\end{array}$} \\
\hline Efectos & \multicolumn{3}{|c|}{$\begin{array}{l}\text { La tala de árboles genera disminución del habitad, erosión } \\
\text { del suelo, disminución de los caudales de agua, contamina- } \\
\text { ción global. }\end{array}$} \\
\hline Impactos ambientales & \multicolumn{3}{|c|}{$\begin{array}{l}\text { Pérdida de flora por el retiro de la cubierta vegetal, gene- } \\
\text { rando alteración en este además del paisaje de la zona de } \\
\text { influencia. }\end{array}$} \\
\hline
\end{tabular}


Tabla 7. Tipos de medidas para la conservación de la flora

\begin{tabular}{|c|c|c|c|c|c|}
\hline \multicolumn{6}{|c|}{ Tipos de medidas: mitigación } \\
\hline $\begin{array}{l}\text { Lugar de } \\
\text { aplicación }\end{array}$ & \multicolumn{5}{|c|}{ Mina de Arcilla Wajira, S.A.S. } \\
\hline \multicolumn{6}{|c|}{ Acciones por desarrollar } \\
\hline \multicolumn{6}{|c|}{$\begin{array}{l}\text { - Realizar revegetalización y reforestación de zonas intervenidas. } \\
\text { - Realizar podas en vez de talas de árboles. } \\
\text { - Reducir la intervención de la flora presente en la zona. }\end{array}$} \\
\hline \multicolumn{6}{|c|}{ Alternativas para realizar las acciones planteadas } \\
\hline \multicolumn{6}{|c|}{$\begin{array}{l}\text { - "Elaboración de la zonificación de las áreas de protección } \\
\text { - Identificación de especies de flora propias del área de influencia del proyecto. } \\
\text { - Realizar un programa de educación ambiental con respecto al conocimiento y la } \\
\text { importancia de protección de la flora" (Avancejuridico.com). }\end{array}$} \\
\hline \multicolumn{6}{|c|}{ Descripción de las medidas } \\
\hline \multicolumn{6}{|c|}{ Mitigación } \\
\hline \multicolumn{6}{|c|}{$\begin{array}{l}\text { Objetivo: Disminuir la tala intensiva en la zona y a su vez evitar posibles afectaciones en } \\
\text { la flora. }\end{array}$} \\
\hline Actividades & $\begin{array}{l}\text { Tiempo de } \\
\text { ejecución } \\
\text { (mes) }\end{array}$ & Responsable & $\begin{array}{l}\text { Recurso } \\
\text { humano }\end{array}$ & $\begin{array}{l}\text { Recurso } \\
\text { físico }\end{array}$ & $\begin{array}{l}\text { Costos } \\
\text { asociados }\end{array}$ \\
\hline $\begin{array}{l}\text { Siembra de } \\
\text { árboles }\end{array}$ & 2 & $\begin{array}{l}\text { Agrónomos y } \\
\text { Supervisor en } \\
\text { reforestación }\end{array}$ & 6 & $\begin{array}{l}200 \text { plán- } \\
\text { tulas }\end{array}$ & $\$ 55000000$ \\
\hline $\begin{array}{l}\text { Mantenimiento } \\
\text { de suelos }\end{array}$ & 2 & & 2 & $\begin{array}{c}\text { Abono, } \\
\text { palas }\end{array}$ & $\$ 3000000$ \\
\hline
\end{tabular}


Tabla 8. Ficha para corregir y mitigar los efectos sobre las aguas subterráneas y superficiales

\begin{tabular}{|c|c|c|c|}
\hline \multirow[t]{2}{*}{ Ficha } & \multirow[t]{2}{*}{ PGS 005} & Programa & $\begin{array}{l}\text { Medidas de correc- } \\
\text { ción y mitigación. }\end{array}$ \\
\hline & & Proyecto & $\begin{array}{l}\text { Determinación de la } \\
\text { calidad de las aguas } \\
\text { subterráneas y super- } \\
\text { ficiales. }\end{array}$ \\
\hline Objetivo & \multicolumn{3}{|c|}{$\begin{array}{l}\text { "Aplicar las medidas de manejo ambiental para la minimiza- } \\
\text { ción de los impactos generados por el manejo inadecuado de } \\
\text { cuerpos de agua, aguas lluvias y escorrentías" (Avancejuridi- } \\
\text { co.com). }\end{array}$} \\
\hline Meta & \multicolumn{3}{|c|}{$\begin{array}{l}\text { Proteger la calidad de las aguas subterráneas y superficiales y } \\
\text { minimizar la erosión por lluvias y escorrentías. }\end{array}$} \\
\hline $\begin{array}{l}\text { Componentes ambien- } \\
\text { tales afectados }\end{array}$ & $\begin{array}{ll}- & \text { Agua } \\
\text { - } & \text { Flora } \\
\text { - } & \text { Fauna }\end{array}$ & $\begin{array}{l}\text { Aspectos am- } \\
\text { bientales }\end{array}$ & Material suspendido \\
\hline $\begin{array}{l}\text { Actividades que ocasio- } \\
\text { nan el impacto }\end{array}$ & \multicolumn{3}{|c|}{$\begin{array}{l}\text { - Arrastre de material sedimentado por escorrentía y aguas } \\
\text { lluvias. } \\
\text { - Transporte interno y externo. } \\
\text { - Beneficio (molienda, clasificación y lavado) }\end{array}$} \\
\hline Efectos & \multicolumn{3}{|c|}{$\begin{array}{l}\text { Modificar las características fisicoquímicas de las aguas subte- } \\
\text { rráneas y superficiales. }\end{array}$} \\
\hline Impactos ambientales & $\begin{array}{ll}- & \mathrm{C} \\
& \mathrm{ci} \\
- & \mathrm{A} \\
- & \mathrm{S}\end{array}$ & $\begin{array}{l}\text { lación de las aguas } \\
\text { n de los parámetro } \\
\text { ación en cuerpos }\end{array}$ & $\begin{array}{l}\text { abterráneas y superfi- } \\
\text { ísicos y químicos. } \\
\text { aguas. }\end{array}$ \\
\hline
\end{tabular}


Tabla 9. Tipos de medidas para corregir y mitigar los efectos sobre las aguas subterráneas y superficiales

\begin{tabular}{|c|c|c|c|c|c|}
\hline \multicolumn{6}{|c|}{ Tipos de medidas: corrección y mitigación } \\
\hline $\begin{array}{l}\text { Lugar de } \\
\text { aplicación }\end{array}$ & \multicolumn{5}{|c|}{ Mina de Arcilla Wajira, S.A.S. } \\
\hline \multicolumn{6}{|c|}{ Acciones por desarrollar } \\
\hline \multicolumn{6}{|c|}{$\begin{array}{l}\text { - Construir canales que minimicen el arrastre de sedimentos. } \\
\text { - No disponer escombros y residuos en ríos. } \\
\text { - No realizar el lavado y mantenimiento de vehículos cercas de los ríos. } \\
\text { - Minimizar la remoción de la cobertura que controla la velocidad del agua de es- } \\
\text { correntía y la producción de sedimentos" (Avancejuridico.com). }\end{array}$} \\
\hline \multicolumn{6}{|c|}{ Alternativa para realizar las acciones planteadas } \\
\hline \multicolumn{6}{|c|}{$\begin{array}{l}\text { - Construcción y mantenimiento de sistemas para aguas de escorrentías y de reten- } \\
\text { ción de sedimentos }\end{array}$} \\
\hline \multicolumn{6}{|c|}{ Descripción de las medidas } \\
\hline \multicolumn{6}{|c|}{ Prevención } \\
\hline \multicolumn{6}{|c|}{$\begin{array}{l}\text { Objetivo: Reducir la cantidad de material sedimentado en el suelo, para evitar una carga } \\
\text { en exceso. }\end{array}$} \\
\hline Actividades & $\begin{array}{l}\text { Tiempo de } \\
\text { ejecución } \\
\text { (mes) }\end{array}$ & $\begin{array}{l}\text { Responsa- } \\
\text { ble }\end{array}$ & $\begin{array}{l}\text { Recurso } \\
\text { humano }\end{array}$ & Recurso físico & $\begin{array}{l}\text { Costos } \\
\text { asociados }\end{array}$ \\
\hline $\begin{array}{l}\text { Evaluación } \\
\text { de parámetros } \\
\text { físico químicos } \\
\text { y bilógicos del } \\
\text { agua }\end{array}$ & 1 & \multirow{2}{*}{$\begin{array}{l}\text { Contratista } \\
\text { experto } \\
\text { para deter- } \\
\text { minación } \\
\text { de pará- } \\
\text { metros a } \\
\text { evaluar }\end{array}$} & 3 & $\begin{array}{l}\text {-Medidor mul- } \\
\text { tiparámetro. } \\
\text {-pHmetro } \\
\text {-Turbidímetro } \\
\text {-Conductíme- } \\
\text { tro }\end{array}$ & $\$ 6700000$ \\
\hline $\begin{array}{l}\text { Monitoreo para } \\
\text { control y pre- } \\
\text { vención }\end{array}$ & $\begin{array}{l}\text { Durante la } \\
\text { vida útil del } \\
\text { proyecto }\end{array}$ & & 2 & $\begin{array}{l}\text {-personal espe- } \\
\text { cializado }\end{array}$ & $\$ 2400000$ \\
\hline
\end{tabular}




\section{Conclusión}

Se concluye que la mayoría de los componentes, tales como el agua, el suelo, la flora (pérdida de biodiversidad y extinción de las especies endémicas), la fauna en la emigración de especies y la disminución de las especies endémicas, estudiados para las fases de construcción y operación, generan impacto moderado, es decir, requieren medidas bien elaboradas. La recuperación de las condiciones iniciales ameritan cierto tiempo y se precisan prácticas de manejo simples.

Además, se destaca que el componente flora, fauna (especies en peligro de extinción) se pueden decir que son impactos irrelevantes, esto quiere decir que la carencia del impacto o la recuperación inmediata tras el cese de la acción pueden requerir tan solo de medidas de protección generales.

De acuerdo con los resultados de la lista de chequeo, se elaboró un plan de manejo ambiental correspondiente a fichas de manejo para prevenir, corregir, mitigar y compensar aquellos impactos generados por la empresa al medio ambiente. Las medidas de manejo ambiental que se propusieron fueron memorias técnicas (descripción, diseños), tratamientos o planteamientos concretos, técnicas y procedimientos de aplicación, costos y cronogramas de ejecución. Como programas y fichas consideradas para las operaciones del Proyecto se crearon las siguientes: I Programa: Mejorar la calidad del suelo; II Programa: Manejo de material particulado; III Programa: Plan de recuperación de la flora; IV Programa: Medidas de corrección y mitigación.

\section{Referencias}

Alcaldía de Medellín (2011). Plan Ambiental Municipal (PAM) 2012-2019 hacia una ciudad sostenible. Tomo II. Diagnóstico actualizado.

Avancejurídico.com. Resolución 1285 de 2015. [Página web] Recuperado de http:// www.avancejuridico.com/actualidad/ documentosoficiales/2015/49535/r_ mads_1258_2015.html

Benito, O. y Huamán, I. (2014). Optimización y modernización en el proceso de obtención de arena de sílice para incrementar la producción en la cantera Santa Rosa 94-I C.C. Llocllapampa. Tesis para optar el título de Ingeniero de Minas, Universidad Nacional del Centro del Perú, Huancayo, Perú.

Etter, A. (1998). Mapa General de Ecosistemas de Colombia (1:2000000). En Ministerio de Ambiente, Vivienda y Desarrollo Terri- 
torial (MAVDT) (2010). Resolución 1023 del 2010.

Ramírez, M. (2008). Sostenibilidad de la explotación de materiales de construcción en el Valle de Aburrá. Tesis de Maestría en Medio Ambiente y Desarrollo, Universidad Nacional de Colombia, Sede Medellín.

Sánchez, J. (2001). El medio ambiente en Colombia: uso del territorio en Colombia. Instituto de Ciencias Naturales. Facultad de Ciencias. Instituto de Estudios Ambientales. Universidad Nacional de Colombia. Instituto de Hidrología, Meteorología y Estudios Ambientales, Ministerio del Medio
Ambiente. República de Colombia. A través de Garzón, N (2013). Análisis preliminar de los impactos ambientales y sociales generados por la minería de arcillas a cielo abierto en la vereda El Mochuelo Bajo, Ciudad Bolívar, Bogotá, D.C., Estudio de caso. Trabajo de grado, Pontificia Universidad Javeriana, Facultad de Estudios Ambientales y Rurales, Bogotá, D. C.

Vega, R. (2007). Los impactos sobre la salud humana de los polvos de minerales y el desarrollo sustentable de la minería como alternativa para mitigar sus efectos. Revista Futuros.info $V(18), 1-16$. 